\title{
CORRELATIONAL STUDY OF THROMBOCYTOPENIA AND BLEEDING COMPLICATIONS IN DENGUE
}

\author{
Srinivasa Kaligonahalli Venkataramanappa1, Hareesh Rangaswamaiah2, Sindhu Nagaraj3
}

${ }^{1}$ Associate Professor, Department of General Medicine, Dr. B. R. Ambedkar Medical College and Hospital. ${ }^{2}$ Associate Professor, Department of General Medicine, Dr. B. R. Ambedkar Medical College and Hospital.

3Postgraduate Student, Department of General Medicine, Dr. B. R. Ambedkar Medical College and Hospital.

\section{ABSTRACT}

\section{BACKGROUND}

Dengue is a viral disease which is acquired through mosquito bites. It is spread over 128 countries globally. Bleeding is one of the complications of Dengue.

Objectives- This study was designed to find the prevalence and type of bleeding in correlation with the platelet count in Dengue.

\section{MATERIALS AND METHODS}

It is a retrospective study and data is collected from records. Information about age, gender, symptoms of bleeding, medications used, investigations done at admission and during the stay in the hospital, management and clinical outcome are collected. Statistical analysis was done to determine the significance of association.

\section{RESULTS}

After exclusion of patients with specific internal bleeding and on drugs like antiplatelets and NSAIDs, 56 patients were studied. 34 were male, 22 were female. NS1 positivity was positively associated with severity of illness. 12 of 14 patients with internal bleeding were NS1 positive. 9 of 14 patients with bleeding had platelet count $<50,000 / \mathrm{mm}^{3}$. Pattern of bleeding- 8 patients had gastrointestinal bleed and 6 had mucocutaneous bleed.

\section{CONCLUSION}

Dengue fever patients can present with bleeding complications more so with severe thrombocytopenia $\left(<50,000 / \mathrm{mm}^{3}\right)$. Prevalence of gastrointestinal bleed and mucocutaneous bleed is more among bleeding complications.

\section{KEYWORDS}

NSAIDs-Non-Steroidal Anti-Inflammatory drugs, NS1-Non-Structural Protein 1, IgG-Immunoglobulin G, IgM-Immunoglobulin M, ARDS-Acute Respiratory Distress Syndrome, ARF-Acute Renal Failure.

HOW TO CITE THIS ARTICLE: Venkataramanappa SK, Rangaswamaiah H, Nagaraj S. Correlational study of thrombocytopenia and bleeding complications in dengue. J. Evolution Med. Dent. Sci. 2017;6(14):1084-1087, DOI: 10.14260/Jemds/2017/235

\section{BACKGROUND}

The incidence of Dengue has grown dramatically around the world in recent decades. The actual number of Dengue cases are underreported and many cases are misclassified. One recent estimate indicates 390 million Dengue infections per year (95\% credible interval 284-528 million) of which 96 million (67-136 million) manifest clinically (with any severity of disease). Another study of prevalence of Dengue estimates that 3.9 billion people in 128 countries are at risk of infection with Dengue viruses.

Based on the data of National Vector Borne Disease Control Program (NVBDCP), the number of cases reported in 2013 was about 74,454 for Dengue with 157 deaths. The data in the website of NVBDCP ${ }^{1}$ and earlier publications of NIV shows that Dengue has been endemic in 16 states. Although the number of cases has shown a steady rise the mortality has reduced. This reduction is probably the result of early diagnosis and better management.

Financial or Other, Competing Interest: None.

Submission 28-11-2016, Peer Review 01-02-2017,

Acceptance 07-02-2017, Published 16-02-2017.

Corresponding Author:

Dr. Srinivasa Kaligonahalli Venkataramanappa,

No .9, I Cross, NMH Layout,

Nagasandra Post, Bengaluru-73.

E-mail: drsrinivas08@gmail.com

DOI: $10.14260 /$ jemds $/ 2017 / 235$

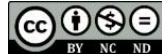

The loss to the economy is 264 disability-adjusted life years (DALY) per million population. Though clear estimates of Dengue are not available from India on account of poor reporting, it appears that Dengue cases are on the rise.

Dengue is a mosquito borne viral disease that has rapidly spread in all regions. Dengue virus is transmitted by female mosquito mainly of the species Aedes aegypti and to a lesser extent Aedes albopictus. There are four distinct but closely related serotypes of the virus that cause Dengue.

Dengue virus was referred in 992 as "water poison" associated with flying insect in a Chinese medical encyclopaedia from the Jin Dynasty (265-420 AD). The word "Dengue" has been derived from the Swahili phrase Kadinga pepo-it means "cramp-like-seizure". In 1943, Ren Kimura and Susumu Hotta, the two scientists of Japan isolated dengue virus from the blood samples of patients residing in Nagasaki, Japan. After 1 year, another two scientists, Albert B and Walter Schlesinger isolated the virus independently.

The genome of DENV is approximately 11,000 base pairs long, with its 5'-cap and 3'-end non-polyadenylated. The genome has a single open-reading frame, which encodes 10 proteins. Seven non-structural (NS) proteins are encoded by the 3'-two thirds of the viral genome: NS1, NS2A, NS2B, NS3, NS4A, NS4B, and NS5. The designation DENV does not refer to a single member of the flavivirus genus but rather to a complex of four closely related viruses, DENV-1, 2, 3, and 4.

Dengue virus primarily is transmitted to humans through an infected mosquito bite, human-to-human transmission 
does not occur. However, DENV can be transmitted from an infected mother to her child during parturition. More research is needed to determine transmission by perinatal transmission, blood transfusion, organ transplantation, needle injury or laboratory accident.

After an incubation period of 4-10 days, infection by any of the four virus serotypes can produce a wide spectrum of illness, although most infections are asymptomatic or subclinical. Primary infection is thought to induce lifelong protective immunity to the infecting serotype. Individuals suffering an infection are protected from clinical illness with a different serotype within 2-3 months of the primary infection but with no long-term cross-protective immunity. Subsequent infection by other serotypes increases the risk of developing severe Dengue.

Dengue haemorrhagic fever is characterised by high fever, haemorrhagic phenomenon, hepatomegaly and shock, moderate to marked thrombocytopenia with concurrent haemoconcentration.

Expanded Dengue Syndrome is with myocarditis, pancreatitis, encephalitis, hepatitis, ARF, ARDS, rhabdomyolysis.

Dengue fever can present with bleeding complications and reinforces the importance of detailed clinical evaluation. Bleeding is the most common complication reported in patients with Dengue fever, particularly adult patients may present with bleeding than children(2)and bleeding manifestation may occur in the absence of plasma leakage.(3) This observation raises questions that bleeding and plasma leakage may be two independent complications. Hence, this study was designed to observe the prevalence of bleeding tendencies in the entire range of platelet count and the prevalence of type of bleed at different platelet count level.

Other important manifestations:

Acute abdomen- Acute abdominal pain is a common symptom in dengue infection. It can be due to hepatitis, acalculous cholecystitis and shock, pancreatitis and occasionally misdiagnosed as acute appendicitis. In patients with shock, the abdominal pain is relieved by intravenous fluid therapy.

Hepatitis and liver failure- Commonest involvement of liver in Dengue is asymptomatic elevation of liver enzymes. But clinically significant hepatitis or hepatic failure may occur in severe Dengue. These can be either due to direct involvement of liver or shock.

\section{MATERIALS AND METHODS}

This was a retrospective study of patients hospitalised with a diagnosis of Dengue disease between May $1^{\text {st }}$ through August 31st 2014 at a Tertiary Care Teaching Hospital, Dr. B. R. Ambedkar Medical College, Bengaluru. Medical records were perused by one of the study investigators to identify records of participants satisfying 2009 WHO evaluation classification for Dengue along with positive serology for acute Dengue infection using IgM, IgG, NS1 ELISA kit.

\section{Data Collection}

Data was collected about the confirmed Dengue cases from their confirmed medical case records. The information about the age, gender, symptoms of bleeding prior and during Hospital stay, medications especially antiplatelets and NSAIDs are collected.

After admission, the following information is collectedsigns of cutaneous and mucosal bleed, haemodynamic assessment, complications, investigation reports, medications and about outcome.

Statistical analysis done using chi-square test and p-value was calculated to determine the significance of association.

\section{RESULTS}

The total study group comprised of data from case files of 56 patients after excluding those patients who had specific causes for internal bleeding and who were on prior medications with antiplatelets, anticoagulants and NSAIDS.

Results -A comprehensive list of our study is mentioned in Table-1.

\begin{tabular}{|c|c|c|c|c|c|}
\hline Sl. No. & Age/Sex & Platelet Count & Serology & Manifestations & Platelet Transfusion \\
\hline 1 & $40 / \mathrm{F}$ & 75000 & IgM Positive & No & No \\
\hline 2 & $22 / \mathrm{M}$ & 88000 & IgM Positive & No & No \\
\hline 3 & $60 / \mathrm{M}$ & 100000 & IgM Positive & No & No \\
\hline 4 & $25 / \mathrm{F}$ & 30000 & IgM Positive & Melaena, Bleeding PV & Yes \\
\hline 5 & $42 / \mathrm{F}$ & 45000 & IgM Positive & Melaena, ARDS & Yes \\
\hline 6 & $18 / \mathrm{M}$ & 45000 & NS1 Positive & Melaena, MODS, Polyserositis, & No \\
\hline 7 & $26 / F$ & 13000 & NS1 Positive & Shock, Polyserositis & No \\
\hline 8 & $40 / \mathrm{M}$ & 80000 & IgG Positive & Loose Stool & No \\
\hline 9 & $52 / \mathrm{M}$ & 25000 & IgG Positive & Frank Blood Per Rectum & Yes \\
\hline 10 & $32 / \mathrm{F}$ & 32000 & NS1 Positive & No & No \\
\hline 11 & $30 / \mathrm{M}$ & 39000 & IgG Positive & No & Yes \\
\hline 12 & $19 / \mathrm{F}$ & 56000 & IgG Positive & No & No \\
\hline 13 & $19 / \mathrm{F}$ & 78000 & IgM Positive & No & No \\
\hline 14 & $32 / \mathrm{F}$ & 66000 & NS1 Positive & No & No \\
\hline 15 & $25 / \mathrm{M}$ & 56000 & IgG Positive & No & No \\
\hline 16 & $15 / \mathrm{F}$ & 90000 & IgM Positive & No & No \\
\hline 17 & $24 / F$ & 50000 & IgG Positive & No & No \\
\hline 18 & $25 / \mathrm{F}$ & 60000 & IgG Positive & No & No \\
\hline 19 & $23 / \mathrm{F}$ & 60000 & IgG Positive & No & No \\
\hline 20 & $27 / \mathrm{M}$ & 50000 & NS1 Positive & No & No \\
\hline 21 & $39 / \mathrm{F}$ & 135000 & IgM Positive & No & No \\
\hline 22 & $26 / \mathrm{M}$ & 90000 & IgM Positive & No & No \\
\hline 23 & $26 / \mathrm{F}$ & 80000 & IgG Positive & No & No \\
\hline
\end{tabular}




\begin{tabular}{|c|c|c|c|c|c|}
\hline 24 & $25 / F$ & 140000 & IgG Positive & No & No \\
\hline 25 & $35 / \mathrm{M}$ & 127000 & IgG Positive & No & No \\
\hline 26 & $35 / \mathrm{M}$ & 50000 & IgG Positive & No & No \\
\hline 27 & $28 / \mathrm{M}$ & 15000 & $\begin{array}{l}\text { IgG Positive, } \\
\text { IgM Positive }\end{array}$ & No & No \\
\hline 28 & $18 / \mathrm{F}$ & 82000 & NS1 Positive & No & No \\
\hline 29 & $35 / \mathrm{M}$ & 104000 & IgM Positive & No & No \\
\hline 30 & $17 / \mathrm{M}$ & 75000 & IgG Positive & No & No \\
\hline 31 & $19 / \mathrm{M}$ & 44000 & IgM Positive & No & No \\
\hline 32 & $31 / \mathrm{F}$ & 77000 & NS1 Positive & No & No \\
\hline 33 & $20 / \mathrm{F}$ & 66000 & IgM Positive & No & No \\
\hline 34 & $44 / \mathrm{M}$ & 48000 & NS1 Positive & Melaena & No \\
\hline 35 & $27 / \mathrm{M}$ & 10000 & IgG Positive & No & No \\
\hline 36 & $34 / \mathrm{M}$ & 72000 & NS1 Positive & No & No \\
\hline 37 & $24 / \mathrm{M}$ & 135000 & IgM Positive & No & No \\
\hline 38 & $70 / \mathrm{M}$ & 109000 & NS1 Positive & No & No \\
\hline 39 & $33 / \mathrm{M}$ & 94000 & NS1 Positive & No & No \\
\hline 40 & $42 / \mathrm{M}$ & 28000 & $\begin{array}{l}\text { IgG Positive, } \\
\text { IgM Positive }\end{array}$ & Melaena & No \\
\hline 41 & $19 / \mathrm{M}$ & 94000 & Negative & Melaena & No \\
\hline 42 & $59 / \mathrm{M}$ & 16000 & NS1 Positive & No & No \\
\hline 43 & $42 / \mathrm{F}$ & 142000 & IgM Positive & Bradycardia & No \\
\hline 44 & $21 / \mathrm{M}$ & 16000 & IgG Positive & Melaena & No \\
\hline 45 & $36 / \mathrm{F}$ & 143000 & IgM Positive & No & No \\
\hline 46 & $18 / \mathrm{M}$ & 96000 & NS1 Positive & No & No \\
\hline 47 & $25 / \mathrm{M}$ & 46000 & IgG Positive & No & No \\
\hline 48 & $30 / \mathrm{M}$ & 34000 & IgM Positive & No & No \\
\hline 49 & $26 / \mathrm{M}$ & 96000 & IgM Positive & No & No \\
\hline 50 & $28 / \mathrm{M}$ & 42000 & IgG Positive & Petechiae Melaena & No \\
\hline 51 & $32 / \mathrm{M}$ & 37000 & NS1 Positive & Bleeding Gums & No \\
\hline 52 & $37 / \mathrm{M}$ & 84000 & IgM Positive & Melaena & No \\
\hline 53 & $32 / \mathrm{F}$ & 6000 & NS1 Positive & Melaena & No \\
\hline 54 & $44 / \mathrm{M}$ & 72000 & IgM Positive & Melaena & No \\
\hline 55 & $39 / \mathrm{M}$ & 66000 & NS1 Positive & Melaena & No \\
\hline 56 & $44 / F$ & 105000 & NS1 Positive & No & No \\
\hline \multicolumn{3}{|c|}{ Total 56} & 13 & 17 & 4 \\
\hline \multicolumn{6}{|c|}{ Table 1} \\
\hline
\end{tabular}

The total study group comprised of data from case files of 56 patients ( 34 male, 22 female) of any age most belonging to age 20-50 years. 13 were found to be positive for Dengue Serology with equal gender representation (7 Male, 6 Female). (6 NS1, 5 IgM and 4 IgG). No particular bleeding predilection was noted towards any of the serology. Bleeding manifestations were found in 14 patients with a male preponderance (11 out 17) and most patients belonging to age 20-40 years. It was found that most patients with platelet counts above $50,000 / \mathrm{mm}^{3}$ did not present or subsequently manifest any bleeding manifestations and hence statistical analysis using chi-square test and $\mathrm{p}$ value was carried out to determine any significance or association.

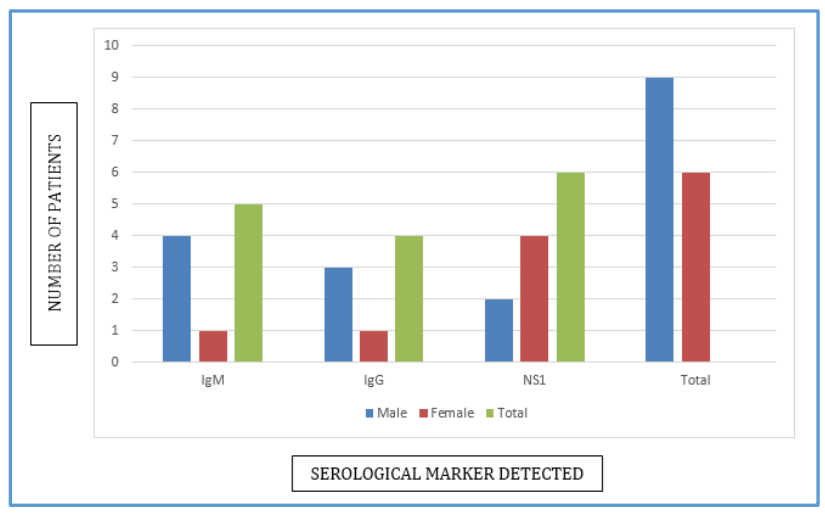

Graph 1
Out of 56 patients, 34 were male and 22 were female in the age group from 20-50 years. When the serology was done NS1 positive was associated positively with the severity of the illness. Out of 14 patients who had bleeding manifestation, 12 were NS1 positive. This finding was statistically significant.

Correlation between platelet count and bleeding manifestation (Table-2):

\begin{tabular}{|c|c|c|c|}
\hline Platelet Count & Yes & No & Grant Total \\
\hline$<50000$ & 9 & 9 & 18 \\
\hline$>50000$ & 5 & 33 & 38 \\
\hline Grand Total & $\mathbf{1 4}$ & $\mathbf{4 2}$ & $\mathbf{5 6}$ \\
\hline \multicolumn{4}{|c|}{ Table 2 } \\
\hline
\end{tabular}

9 of the patients who had bleeding manifestations had platelet count less than $50,000 / \mathrm{mm}^{3}$ during the episode when compared to 5 who had platelet count above $50,000 / \mathrm{mm}^{3}$. A p-value of 0.0004 was determined and significance was noted at $1 \%$.

The pattern of bleeding tendency- about 8 patients had GI bleed and 6 patients had mucocutaneous bleeding. All the patients who had GI bleeding were found to have platelet count less than $50,000 / \mathrm{mm}^{3}$

Among all the patients, 4 patients were given platelet concentrate transfusion. In the presence of bleeding 
Manifestations, $30,000 / \mathrm{mm}^{3}$ of platelet count was considered as the threshold for transfusion. Patients with platelet count above $30,000 / \mathrm{mm}^{3}$ even in presence of melaena and cutaneous bleed were clinically monitored without transfusion. All of them had spontaneous recovery. One patient had a mortality as the patient was diagnosed with Expanded Dengue Syndrome with ARDS.

\section{DISCUSSION}

Emmanuel Bhaskar et al in their study done at Chennai observed $24.1 \%$ overall prevalence of bleeding tendencies in patients with Dengue disease. (39. 8\% in Dengue with severe thrombocytopenia and $14.2 \%$ in Dengue with moderate thrombocytopenia). Gastrointestinal bleeding (33.1\%) was as common as mucocutaneous bleeding (33.7\%).

Shiv Balan et al in their article "predictors of spontaneous bleeding in Dengue" observed that bleeding tendencies correlate with platelet count $<50,000 / \mathrm{mm}^{3}$

In the study, we observed $25 \%$ of overall bleeding tendency out of 56 Dengue patients. Among patients with bleeding manifestations, $64.2 \%$ patients had severe thrombocytopenia i.e. platelet count $<50,000 / \mathrm{mm}^{3}$

This observation highlights that patients with Dengue fever can present with bleeding complications and reinforce the importance of a detailed clinical evaluation in order to deliver appropriate care.

Bleeding complication is the most common complication reported in patients with Dengue fever. Particularly, adult patients may present with bleeding than children and bleeding manifestation may occur in the absence of plasma leakage.(4) This observation raises 2 questions that bleeding and plasma leakage may be 2 independent complications. Thrombocytopenia is strongly associated with bleeding. Many factors contribute to onset of thrombocytopenia, reactive immune response against platelets to decreased platelet production. $(5,6)$

Our results have demonstrated that cases with Dengue fever can manifest with spontaneous bleeding accompanied with changes in peripheral blood count, coagulation and fibrinolysis that may not be significantly detected in Dengue without complication. Dengue fever represents a clinically heterogeneous group of patients and also raise the hypothesis that different pathological mechanisms may be present in these patients.

\section{CONCLUSION}

Dengue fever patients can present with bleeding complications more so with severe thrombocytopenia $\left(<50,000 / \mathrm{mm}^{3}\right)$. The prevalence of GI bleed and mucocutaneous bleed is more among bleeding complications. This reinforces the importance of clinical evaluation and a close monitoring in Dengue fever with bleeding complications. This observation hints at the possible different pathological mechanisms for bleeding and plasma leakage in Dengue fever.

\section{REFERENCES}

[1] 1 National Vector Borne Disease Control ProgrammeDengue/Dengue Hemorrhagic Fever 2013.

http//www. nhe. gov. in/nvbdcp

[2] Hammond SN, Balmaseda A, Perez L, et al. Differences in dengue severity in infants, children, and adults in a 3year hospital-based study in Nicaragua. Am J Trop Med Hyg 2005;73(6):1063-70.

[3] Wichmann O, Hanzsiriwon S, Bowonwatanuwong C, et al. Risk factors and clinical features associated with severe dengue infection in adults and children during 2001 epidemic in Chonburi, Thailand. Trop Med Int Health 2004;9(9):1022-9.

[4] Shivabalan S, Anandnathan K, Balasubramanian S, et al. Predictors of spontaneous bleeding in dengue. Indian J Pediatr 2004;71(1):33-6.

[5] Wang S, He R, Patarapotikul J, et al. Antibody enhanced binding of dengue 2 virus to human platelets. Virology 1995;213(1):254-7.

[6] Orsi FA, Angerami RN, Mazetto BM, et al. Reduced thrombin and excessive fibrinolysis are associated with bleeding complications in patients with dengue fever: a case control study comparing dengue fever-patients with and without bleeding manifestations. BMC Infect Dis 2013;13:350. 